\title{
Partition properties of subsets of $\mathcal{P}_{\kappa} \lambda$
}

\author{
by
}

\author{
Masahiro Shi oy a (Tsukuba)
}

\begin{abstract}
Let $\kappa>\omega$ be a regular cardinal and $\lambda>\kappa$ a cardinal. The following partition property is shown to be consistent relative to a supercompact cardinal: For any $f: \bigcup_{n<\omega}[X]_{\subset}^{n} \rightarrow \gamma$ with $X \subset \mathcal{P}_{\kappa} \lambda$ unbounded and $1<\gamma<\kappa$ there is an unbounded $Y \subset X$ with $\left|f^{\prime \prime}[Y]^{n} \subset\right|=1$ for any $n<\omega$.
\end{abstract}

Let $\kappa$ be a regular cardinal $>\omega, \lambda$ a cardinal $\geq \kappa$ and $F$ a filter on $\mathcal{P}_{\kappa} \lambda$. Partition properties of the form $\mathcal{P}_{\kappa} \lambda \rightarrow\left(F^{+}\right)_{2}^{2}$ (see below for the definition) were introduced by Jech [6]. The case where $F$ is the club filter $\mathcal{C}_{\kappa \lambda}$ was particularly studied in connection with a supercompact cardinal: Menas [14] proved $\mathcal{P}_{\kappa} \lambda \rightarrow\left(\mathcal{C}_{\kappa \lambda}^{+}\right)_{2}^{2}$ for a $2^{\lambda^{<\kappa}}$-supercompact $\kappa$ via a normal ultrafilter $U$ with $\mathcal{P}_{\kappa} \lambda \rightarrow\left(U^{+}\right)_{2}^{2}$. As noted by Kamo [9], Menas' argument can be modified to give the partition property of $\mathcal{P}_{\kappa} \lambda$ for $\kappa$ just $\lambda$-supercompact. For the converse direction Di Prisco and Zwicker [4] and others refined the global result of Magidor [12]: The partition property of $\mathcal{P}_{\kappa} 2^{\lambda^{<\kappa}}$ implies that $\kappa$ is $\lambda$-supercompact.

In [8] Johnson introduced properties of the form $X \rightarrow\left(F^{+}\right)_{2}^{2}$ for $X \in F^{+}$, which means that for any $f:[X]_{\subset}^{2} \rightarrow 2$ there is $Y \in F^{+}$with $Y \subset X$ and $\left|f^{\prime \prime}[Y]_{\subset}^{2}\right|=1$, as well as $F^{+} \rightarrow\left(F^{+}\right)_{2}^{2}$, which means $X \rightarrow\left(F^{+}\right)_{2}^{2}$ for any $X \in F^{+}$. Abe [1] asked whether $\mathcal{F}_{\kappa \lambda}^{+} \rightarrow\left(\mathcal{F}_{\kappa \lambda}^{+}\right)_{2}^{2}$ would fail in ZFC, where $\mathcal{F}_{\kappa \lambda}$ denotes the minimal fine filter on $\mathcal{P}_{\kappa} \lambda$.

In this note we answer the question of Abe:

TheOREM. Let $\kappa$ be a supercompact cardinal and $\lambda$ a cardinal $>\kappa$. Then there is a $\kappa^{+}$-c.c. poset forcing that $\kappa$ is supercompact and $\mathcal{F}_{\kappa \lambda}^{+} \rightarrow\left(\mathcal{F}_{\kappa \lambda}^{+}\right)_{\gamma}^{<\omega}$ for any $1<\gamma<\kappa$.

1991 Mathematics Subject Classification: 03E05, 03E55.

The author enjoyed Prof. Kanamori's hospitality while staying at Boston University as a Japanese Overseas Research Fellow. He also wishes to thank Prof. Abe for helpful conversations, and the referee for improving the presentation. 
Here $F^{+} \rightarrow\left(F^{+}\right)_{\gamma}^{<\omega}$ means that for any $f: \bigcup_{n<\omega}[X]_{\subset}^{n} \rightarrow \gamma$ with $X \in F^{+}$ there is $Y \in F^{+}$with $Y \subset X$ and $\left|f^{*}[Y]_{\subset}^{n}\right|=1$ for any $n<\omega$. Note that $\kappa$ is Ramsey iff $\mathcal{F}_{\kappa \kappa}^{+} \rightarrow\left(\mathcal{F}_{\kappa \kappa}^{+}\right)_{\gamma}^{<\omega}$ for any $1<\gamma<\kappa$.

We generally follow the terminology of Kanamori [10] with the following exception: For a cardinal $\mu \geq \omega$ we set $[X]^{\mu}=\{x \subset X:|x|=\mu\},[X]^{<\mu}=$ $\{x \subset X:|x|<\mu\}$ and $\lim A=\{\alpha<\mu: \sup (A \cap \alpha)=\alpha>0\}$ for $A \subset \mu$. We understand $\bigcup a \subsetneq \bigcap b$ whenever the union $a \cup b$ of $a \in\left[\mathcal{P}_{\kappa} \lambda\right]_{\subset}^{m}$ and $b \in\left[\mathcal{P}_{\kappa} \lambda\right]_{\subset}^{n}$ with $m, n<\omega$ is formed.

We first give two negative partition results, which motivated Abe's question. In [1] Abe proved $\mathcal{F}_{\kappa \lambda}^{+} \nrightarrow\left(\mathcal{F}_{\kappa \lambda}^{+}\right)_{2}^{2}$ under $\lambda^{<\kappa}=2^{\lambda}$. On the other hand, Matet [13], extending a result of Laver (see [7]), got the same conclusion from the opposite assumption:

Proposition 1. Assume $\lambda^{\kappa}=\lambda$. Then $\mathcal{F}_{\kappa \lambda}^{+} \nrightarrow\left(\mathcal{F}_{\kappa \lambda}^{+}\right)_{2}^{2}$.

Proof. First set $\mathcal{P}_{\kappa} \lambda=\left\{x_{\xi}: \xi<\lambda\right\}$ and $\left[\mathcal{P}_{\kappa} \lambda\right]^{\kappa}=\left\{Y_{\alpha}: \alpha<\lambda\right\}$. By induction on $\xi<\lambda$ we construct $z_{\xi} \in \mathcal{P}_{\kappa} \lambda$ and $\left\{y_{\xi}^{\alpha i}: \alpha \in z_{\xi} \wedge i<2\right\}$ so that $x_{\xi} \subset z_{\xi}, z_{\xi} \neq z_{\zeta}, y_{\xi}^{\alpha i} \in Y_{\alpha}, y_{\xi}^{\alpha i} \subsetneq z_{\xi}$ and $y_{\xi}^{\alpha 0} \neq y_{\xi}^{\beta 1}$ for any $\zeta<\xi, i<2$ and $\alpha, \beta \in z_{\xi}$ as follows: At stage $\xi<\lambda$ by induction on $n<\omega$ build $z_{\xi n} \in \mathcal{P}_{\kappa} \lambda$ and $\left\{y_{\xi}^{\alpha i}: \alpha \in z_{\xi n} \wedge i<2\right\}$ so that $x_{\xi} \subset z_{\xi 0} \not \subset \bigcup_{\zeta<\xi} z_{\zeta}, y_{\xi}^{\alpha i} \in Y_{\alpha}, y_{\xi}^{\alpha 0} \neq y_{\xi}^{\beta 1}$ and $z_{\xi n} \cup \bigcup\left\{y_{\xi}^{\alpha i}: \alpha \in z_{\xi n} \wedge i<2\right\} \subsetneq z_{\xi n+1}$. Finally set $z_{\xi}=\bigcup_{n<\omega} z_{\xi n}$. We claim that $f$ defined by $f\left(\left\{y_{\xi}^{\alpha i}, z_{\xi}\right\}\right)=i$ witnesses $\left\{z_{\xi}: \xi<\lambda\right\} \not \rightarrow\left(\mathcal{F}_{\kappa \lambda}^{+}\right)_{2}^{2}$.

Fix an unbounded set $X \subset\left\{z_{\xi}: \xi<\lambda\right\}$. We show $f^{\text {" }}[X]_{\subset}^{2}=2$. Take $\alpha<\lambda$ with $Y_{\alpha} \in[X]^{\kappa}$, and $\xi<\lambda$ with $\alpha \in z_{\xi} \in X$. Then $f\left(\left\{y_{\xi}^{\alpha i}, z_{\xi}\right\}\right)=i$ for $i<2$ by definition, as desired.

The above proof yields in fact for any $\gamma<\kappa$ an unbounded set $X \subset \mathcal{P}_{\kappa} \lambda$ and $f:[X]_{\subset}^{2} \rightarrow \gamma$ such that $f^{\prime \prime}[Y]_{\subset}^{2}=\gamma$ for any unbounded $Y \subset X$.

The analogous problem for the club filter has been solved by Abe [2] via an extension of Magidor's theorem [12]: $\mathcal{C}_{\kappa \lambda}^{+} \not \rightarrow\left(\mathcal{C}_{\kappa \lambda}^{+}\right)_{2}^{2}$. Let us give a canonical witness to his observation by appealing to Magidor's idea more directly:

Proposition 2. Let $\mu<\kappa$ be regular. Then $\left\{x \in \mathcal{P}_{\kappa} \lambda: \operatorname{cf}(x \cap \kappa)=\mu\right\}$ $\not\left(\mathcal{C}_{\kappa \lambda}^{+}\right)_{2}^{2}$.

Proof. Set $S=\left\{x \in \mathcal{P}_{\kappa} \lambda: \operatorname{cf}(x \cap \kappa)=\mu\right\}$ and for $x \in S$ fix an unbounded set $c_{x} \subset x \cap \kappa$ of order type $\mu$. For $\{x, y\} \in[S]_{\subset}^{2}$ let $f(\{x, y\})$ be 0 when $\min \left(c_{x} \Delta c_{y}\right) \in c_{x}$, and 1 otherwise. Fix a stationary set $T \subset S$. We show $f^{\text {" }}[T]_{\subset}^{2}=2$.

First, we have $\gamma<\kappa$ such that for any $w \in \mathcal{P}_{\kappa} \lambda$ there are $w \subset x, y \in T$ with $\gamma \in c_{x}-c_{y}$ : Let $g: \kappa \rightarrow \mathcal{P}_{\kappa} \lambda$ witness the contrary, i.e. $\gamma \in c_{x}$ iff $\gamma \in c_{y}$ for any $\gamma<\kappa$ and $g(\gamma) \subset x, y \in T$. Take $x, y \in C(g) \cap T$ with $x \cap \kappa<y \cap \kappa$ 
by the stationarity of $\{z \cap \kappa: z \in C(g) \cap T\}$ in $\kappa$. Then $c_{x}=c_{y} \cap x \cap \kappa$ has order type $\mu$, contradicting the choice of $c_{y}$.

Now, let $\gamma<\kappa$ be minimal as above. Then for $\alpha<\gamma$ we have $w_{\alpha} \in \mathcal{P}_{\kappa} \lambda$ such that $\alpha \in c_{x}$ iff $\alpha \in c_{y}$ for any $w_{\alpha} \subset x, y \in T$. Set $w=\bigcup_{\alpha<\gamma} w_{\alpha} \in \mathcal{P}_{\kappa} \lambda$. Take $w \subset x \subset y \subset z$ from $T$ with $\gamma \in c_{x} \cap c_{z}-c_{y}$. Then $\min \left(c_{x} \Delta c_{y}\right)=$ $\min \left(c_{y} \Delta c_{z}\right)=\gamma$ by $w_{\alpha} \subset x \subset y \subset z$ for any $\alpha<\gamma$, and hence $f(\{x, y\})=0$ and $f(\{y, z\})=1$ by definition, as desired.

The rest of the paper is devoted to establishing our Theorem. We refer to Baumgartner's expository paper [3] for the rudiments of iterated forcings. We call a poset $\kappa$-centered closed when any centered subset of size $<\kappa$ has a lower bound.

Assume for the moment that $\kappa$ is a compact cardinal and $\lambda \leq 2^{\kappa}$. Fix a coloring $f: \bigcup_{n<\omega}[S]_{\subset}^{n} \rightarrow \gamma$ with $S \subset \mathcal{P}_{\kappa} \lambda$ unbounded and $1<\gamma<\kappa$. Our definition of the poset $Q_{f}$ below owes much to Galvin (see [7]), who proved under $\operatorname{MA}(\lambda)$ that for any $f:[X]_{\subset}^{2} \rightarrow 2$ with $X \subset[\lambda]^{<\omega}$ cofinal there is a cofinal $Y \subset X$ with $\left|f "[Y]_{\subset}^{2}\right|=1$.

Fix a fine ultrafilter $U$ on $S$ and define inductively a $\kappa$-complete ultrafilter $U_{n}$ on $[S]_{\subset}^{n}$ by $U_{0}=\{\{\emptyset\}\}$ and $U_{n+1}=\left\{X:\left\{x:\{a:\{x\} \cup a \in X\} \in U_{n}\right\} \in U\right\}$. For $n<\omega$ let $\beta_{n}$ be the unique $\beta<\gamma$ with $\left\{a \in[S]_{\subset}^{n}: f(a)=\beta\right\} \in U_{n}$. Let $Q_{f}=\left\{p \in[S]^{<\kappa}: \forall m, n<\omega \forall a \in[p]_{\subset}^{m}\left(\left\{b \in[S]_{\subset}^{n}: f(a \cup b)=\beta_{m+n}\right\} \in U_{n}\right)\right\}$, and $q \leq p$ iff $q \supset p$ and $y \not \subset x$ for any $x \in p$ and $y \in q-p$. Let us observe some basic properties of $Q_{f}$.

First, for a generic filter $G \subset Q_{f}, \bigcup G$ is unbounded in $\mathcal{P}_{\kappa} \lambda$ by the density of $\left\{q \in Q_{f}: \exists y \in q(x \subset y)\right\}$ for any $x \in \mathcal{P}_{\kappa} \lambda$, and homogeneous for $f: f$ " $[\bigcup G]_{\subset}^{n}=\left\{\beta_{n}\right\}$ for any $n<\omega$.

Next, we have the $\kappa$-centered closure of $Q_{f}: \bigcup D$ is a lower bound of a centered set $D \in\left[Q_{f}\right]^{<\kappa}$.

Finally, we invoke an argument of Engelking and Karłowicz [5] to show that $Q_{f}$ is $\kappa$-linked. Fix an injection $\pi: \mathcal{P}_{\kappa} \lambda \rightarrow{ }^{\kappa} 2$. For $A \subset{ }^{\alpha} 2$ with $\alpha<\kappa$ set $Q_{f, A}=\left\{p \in Q_{f}:\{\pi(x) \mid \alpha: x \in p\}=A \wedge\left\langle\pi(z) \mid \alpha: z \in \bigcup_{x \in p} \mathcal{P} x\right\rangle\right.$ is injective $\}$. Then $Q_{f}=\bigcup\left\{Q_{f, A}: \exists \alpha<\kappa\left(A \subset{ }^{\alpha} 2\right)\right\}$ by the inaccessibility of $\kappa$. To see that $Q_{f, A}$ is linked, fix $p, q \in Q_{f, A}$. Then $x \not \subset y$ for any $x \in p-q$ and $y \in q$ : Otherwise we would have $x=z$ for some $x \in p-q, y \in q$ with $x \subset y$ and $z \in q$ with $\pi(x)|\alpha=\pi(z)| \alpha$. Similarly, $y \not \subset x$ for any $x \in p$ and $y \in q-p$. Thus $p \cup q \leq p, q$, as desired.

Before starting the proof of our Theorem, we need to generalize a result of Baumgartner [3]:

Lemma. Assume $2^{<\kappa}=\kappa$. Let $\left\langle P_{\alpha}, \dot{Q}_{\alpha}: \alpha<\beta\right\rangle$ be a $<<$-support iteration such that $\Vdash_{\alpha}$ " $\dot{Q}_{\alpha}$ is $\kappa$-centered closed and $\kappa$-linked" for any $\alpha<\beta$. Then $P_{\beta}$ is $\kappa$-directed closed and $\kappa^{+}$-c.c. 
Pr o o f. It is easily seen that the $\kappa$-centered closure implies the $\kappa$-directed closure, which is preserved by $<\kappa$-support iterations.

To see the $\kappa^{+}$-c.c., fix $X \in\left[P_{\beta}\right]^{\kappa^{+}}$. For $\alpha<\beta$ let $\Vdash_{\alpha}$ " $\dot{Q}_{\alpha}=\bigcup_{\gamma<\kappa} \dot{Q}_{\alpha \gamma}$ with $\dot{Q}_{\alpha \gamma}$ linked for any $\gamma<\kappa$ ". For $p \in X$ by induction on $\xi<\kappa$ build $p_{\xi} \leq p, \alpha_{\xi}^{p} \in \operatorname{supp}\left(p_{\xi}\right)$ and $\gamma_{\xi}^{p}<\kappa$ so that $p_{\xi} \leq p_{\zeta}$ for any $\zeta<\xi$, $p_{\xi+1} \mid \alpha_{\xi}^{p} \Vdash_{\alpha_{\xi}^{p}}$ " $p_{\xi}\left(\alpha_{\xi}^{p}\right) \in \dot{Q}_{\alpha_{\xi}^{p} \gamma_{\xi}^{p}}$ ", and $\left\{\xi<\kappa: \alpha_{\xi}^{p}=\alpha\right\}$ is unbounded for any $\alpha \in \bigcup_{\zeta<\kappa} \operatorname{supp}\left(p_{\zeta}\right)$. Take $Y \in[X]^{\kappa^{+}}$and $\delta<\kappa$ so that $\delta \in$ $\Delta_{\zeta<\kappa} \bigcap\left\{\lim \left\{\xi<\kappa: \alpha_{\xi}^{p}=\alpha\right\}: \alpha \in \operatorname{supp}\left(p_{\zeta}\right)\right\}$ for any $p \in Y$. Note that $\left\{\alpha_{\xi}^{p}: \xi<\delta\right\}=\bigcup_{\zeta<\delta} \operatorname{supp}\left(p_{\zeta}\right)$ for any $p \in Y$. Next take $Z \in[Y]^{\kappa^{+}}$so that $\left\{\left\{\alpha_{\xi}^{p}: \xi<\delta\right\}: p \in Z\right\}$ forms a $\Delta$-system with root $d \in[\beta]^{<\kappa}$. Finally, take $W \in[Z]^{\kappa^{+}}$and $H \in[\delta \times d \times \kappa]^{<\kappa}$ so that $\left\{\left(\xi, \alpha_{\xi}^{p}, \gamma_{\xi}^{p}\right): \xi<\delta \wedge \alpha_{\xi}^{p} \in d\right\}=H$ for any $p \in W$. We show that $W$ is linked, as desired.

Fix $p, q \in W$. Inductively we build a lower bound $r \in P_{\beta}$ of $\left\{p_{\xi}: \xi<\delta\right\}$ $\cup\left\{q_{\xi}: \xi<\delta\right\}$ with support $\bigcup_{\zeta<\delta} \operatorname{supp}\left(p_{\zeta}\right) \cup \bigcup_{\zeta<\delta} \operatorname{supp}\left(q_{\zeta}\right)$. At stage $\alpha<\beta$ we claim that $\left\{\xi<\delta: r \mid \alpha \Vdash_{\alpha} " p_{\xi}(\alpha) \| q_{\xi}(\alpha)\right.$ " $\}$ is unbounded, which implies $r \mid \alpha \Vdash_{\alpha}$ " $\left\{p_{\xi}(\alpha): \xi<\delta\right\} \cup\left\{q_{\xi}(\alpha): \xi<\delta\right\}$ is centered", as desired, since $r \mid \alpha \Vdash_{\alpha}$ " $\left\{p_{\xi}(\alpha): \xi<\delta\right\}$ and $\left\{q_{\xi}(\alpha): \xi<\delta\right\}$ are descending". Let us concentrate on the nontrivial case where $\alpha \in d=\bigcup_{\zeta<\delta} \operatorname{supp}\left(p_{\zeta}\right) \cap \bigcup_{\zeta<\delta} \operatorname{supp}\left(q_{\zeta}\right)$.

Fix $\xi<\delta$ with $\alpha_{\xi}^{p}=\alpha$. Then $r\left|\alpha \leq p_{\xi+1}\right| \alpha, q_{\xi+1} \mid \alpha$ forces " $p_{\xi}(\alpha), q_{\xi}(\alpha) \in$ $\dot{Q}_{\alpha \gamma}$ ", where $(\xi, \alpha, \gamma) \in H$. Now the claim follows, since $\left\{\xi<\delta: \alpha_{\xi}^{p}=\alpha\right\}$ is unbounded by the choice of $\delta$.

Proof of Theorem. First, we force with the Laver poset [11] for $\kappa$ and then add $\lambda$ Cohen subsets of $\kappa$ to ensure that $\kappa$ is supercompact and $\lambda \leq 2^{\kappa}$ in the further extensions. Next, we perform a $<\kappa$-support iteration $\left\langle P_{\alpha}, \dot{Q}_{\alpha}: \alpha<2^{\lambda^{<\kappa}}\right\rangle$ with $\Vdash_{\alpha}$ " $\dot{Q}_{\alpha}=Q_{\dot{f}}$ " for some canonical $P_{\alpha}$-name $\dot{f}$ for a coloring. The standard inductive argument, together with the $\kappa$-closure and the $\kappa^{+}$-c.c. of $P_{\alpha}$, shows that for any $\alpha<2^{\lambda^{<\kappa}}, P_{\alpha}$ is of size $\leq 2^{\lambda^{<\kappa}}$, and so is the set of canonical $P_{\alpha}$-names for colorings, whose union can be identified with that of canonical $P_{2^{\lambda}<\kappa}$-names for colorings. Thus the iteration can be arranged so that a homogeneous set for a coloring in the final model by $P_{2^{\lambda}<\kappa}$ appears in an intermediate model, which, by absoluteness of $\mathcal{P}_{\kappa} \lambda$, remains unbounded, as desired.

\section{References}

[1] Y. Abe, Combinatorics for small ideals on $\mathcal{P}_{\kappa} \lambda$, Math. Logic Quart. 43 (1997), 541-549.

[2] - private communication. 
[3] J. Baumgartner, Iterated forcing, in: Surveys in Set Theory, A. Mathias (ed.), London Math. Soc. Lecture Note Ser. 87, Cambridge Univ. Press, Cambridge, 1983, $1-59$.

[4] C. Di Prisco and W. Zwicker, Flipping properties and supercompact cardinals, Fund. Math. 109 (1980), 31-36.

[5] R. Engelking and M. Karłowicz, Some theorems of set theory and their topological consequences, ibid. 57 (1965), 275-285.

[6] T. Jech, Some combinatorial problems concerning uncountable cardinals, Ann. Math. Logic 5 (1973), 165-198.

[7] T. Jech and S. Shelah, A partition theorem for pairs of finite sets, J. Amer. Math. Soc. 4 (1991), 647-656.

[8] C. Johnson, Some partition relations for ideals on $\mathcal{P}_{\kappa} \lambda$, Acta Math. Hungar. 56 (1990), 269-282.

[9] S. Kamo, Ineffability and partition property on $\mathcal{P}_{\kappa} \lambda$, J. Math. Soc. Japan 49 (1997), $125-143$.

[10] A. Kanamori, The Higher Infinite, Springer, Berlin, 1994.

[11] R. Laver, Making the supercompactness of $\kappa$ indestructible under $\kappa$-directed closed forcing, Israel J. Math. 29 (1978), 385-388.

[12] M. Magidor, Combinatorial characterization of supercompact cardinals, Proc. Amer. Math. Soc. 42 (1974), 279-285.

[13] P. Matet, handwritten notes.

[14] T. Menas, A combinatorial property of $\mathcal{P}_{\kappa} \lambda$, J. Symbolic Logic 41 (1976), 225-234.

Institute of Mathematics

University of Tsukuba

Tsukuba, 305-8571 Japan

E-mail: shioya@math.tsukuba.ac.jp 